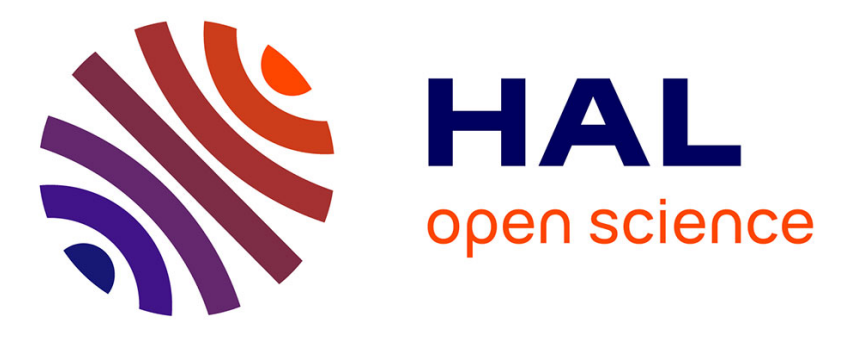

\title{
Automatic brain tumor extraction using fuzzy information fusion
}

Weibei Dou, Qingmin Liao, Su Ruan, Daniel Bloyet, Jean-Marc Constans, Yanping Chen

\section{- To cite this version:}

Weibei Dou, Qingmin Liao, Su Ruan, Daniel Bloyet, Jean-Marc Constans, et al.. Automatic brain tumor extraction using fuzzy information fusion. 2nd International Conference on Image and Graphic (ICIG 2002), 2002, Hefei, China. pp.604-609, 10.1117/12.477203 . hal-01069571

\section{HAL Id: hal-01069571 https://hal.science/hal-01069571}

Submitted on 30 Sep 2014

HAL is a multi-disciplinary open access archive for the deposit and dissemination of scientific research documents, whether they are published or not. The documents may come from teaching and research institutions in France or abroad, or from public or private research centers.
L'archive ouverte pluridisciplinaire HAL, est destinée au dépôt et à la diffusion de documents scientifiques de niveau recherche, publiés ou non, émanant des établissements d'enseignement et de recherche français ou étrangers, des laboratoires publics ou privés. 


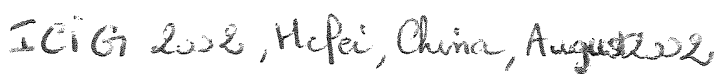

$$
\begin{aligned}
& \text { Plx. SPiE-Vol. } 475 \text {-pp. 604-609 }
\end{aligned}
$$

\title{
Automatic brain tumor extraction using fuzzy information fusion
}

\author{
Weibei DOU ${ }^{* a}$, Qingmin LIAO $^{\mathrm{a}}$, Su Ruan ${ }^{* * b}$, Daniel Bloyet ${ }^{\mathrm{b}}$ \\ Jean-Mac CONSTANS ${ }^{c}$, Yanping CHEN $^{d}$ \\ ${ }^{a}$ Department of Electronic Engineering, Tsinghua University, Beijing China \\ ${ }^{\mathrm{b}}$ GREYC-ISMRA, CNRS UMR 6072, Caen France \\ 'Unite d' IRM, CHRU, Caen France \\ ${ }^{\mathrm{d} I m a g i n g}$ Diagnostic Center, Nanfang Hospital, Guangzhou China
}

\begin{abstract}
This paper presents a fuzzy information fusion method to automatically extract tumor areas of human brain from multispectral magnetic resonance (MR) images. The multispectral images consist of $T 1$-weighted (T1), proton density (PD), and $T 2$-weighted (T2) feature images, in which signal intensities of a tumor are different. Some tissue is more visible in one image type than the others. The fusion of information is therefore necessary. Our method, based on the fusion of information, model the fuzzy information about the tumor by membership functions. This modelisation is based on the a priori knowledge of radiology experts and the MR signals of the brain tissues. Three membership functions related to the three images types are proposed according to their characteristics. The brain extraction is then carried out by using the fusion of all three fuzzy information. The experimental results (based on 5 patients studied) show a mean false-negative of $2 \%$ and a mean false-positive of $1.3 \%$, comparing to the results obtained by a radiology using manual tracing.
\end{abstract}

Keywords: magnetic resonance imaging (MRI), tumor, information fusion, fuzzy

\section{INTRODUCTION}

Magnetic resonance imaging (MRI) is widely used in modern medicine to study the organic function, to diagnostic lumors. to advise treatments and to assist operations etc. MRI can provide a variety of information about brain tissues as a function of the excitation sequence. The tissue abnormalities can be found also through a variety of MR feature images. The study of brain using MR imaging is one of very important fundamental research topic in human science. There is a list of several published researches of brain based on MR images in [1]. Brain tumor can be considered as one of abnormality tissue. The segmentation of brain tumor allows us to obtain the information about the size, the position of tumors, the sort, and the relationship between tumor and surrounding tissues. It provides a very powerful assistant of medicine for following his treatment to appraise the therapies. For image guided surgery, it can be used also to aid operators to select the route of operation to avoid blood vessel and some sensitive and important tissues. So the accuracy of extraction is absoluteness important target. The automatism, robustness and speediness of tumor extraction are also important for application. A knowledge-based brain tumor extraction technique is presented in [2]. An anatomic brain phantom is used to evaluate the accuracy of tumor description by using multimodality image fusion technique of Positron emission tomography (PET) and MRI/CT images [3]. In view of the ambiguous, complementary and redundant characters of MR images, the fuzzy logic and information fusion techniques are selected firstly in brain tissue research [1][4]. The FuzzyConnectedness is used successfully to quantify the multiple sclerosis lesion [4].

douwbalee.tsinghua.edu.cn: phone (+86 10 62781703); fax (+86 10 62770317); Department of Electronic Engineering, Tsinghua Iniversity. 100084 Beijing. China. 
In this paper, we propose a new method of brain tumor extraction. The goal of our research is to extract automatically and correctly the brain tumor using T1, T2 and PD MR feature images. The tumor area is extracted through fusion of the fuzzy sets characterized by membership functions of tumor of T1, T2 and PD feature image. The membership function is calculated from the descriptions of the experts about tumors. Our method consists of four steps, described as follows. The first step is aimed to align the $T 2$ and PD volumes into the $T 1$ volume using a linear registration method presented in [5]. Since the resolution of $\mathrm{T} 1$ images is better than others types of images, the brain tissue segmentation is carried out only on T1 images. The segmentation method using fuzzy Markovian Random Fields published in [6] is used here to segment the brain into three principal brain tissues (white matter, gray matter and Cerebro-spinal fluid (CSF)), called pure classes, and their mixtures, called mixclasses. Each mixclasse has two components which describe the mix percentages of two brain tissues. The sum of them is equal to 1 . The voxels being in the same class and connect are labeled as a same region. A set of regions are then obtained. These regions on $\mathrm{T} 1 \mathrm{images}$ are then projected onto the other types of registered images. The third step is to extract separately the information about the means and the variance of each region on T1, T2 and PD, in order to fuzzily classifier them to the tumor. The membership function of the fuzzy sets related to the tumor on T1, T2 and PD are differently modeled according to characteristics of each type of image and the a priori knowledge of radiology experts. Finally the brain tumor is extracted from the set of regions obtained in step 2 by using the fusion of the fuzzy information obtained in the step 3.

\section{FUZZY MODELIDATIONS BASED ON BRAIN TUMOR KNOWLEDGE}

From the point of view of fuzzy set and fuzzy information theory, the brain tumor area visualized in MR images can be considered as a fuzzy set, due to the noise, partial volume effects and the image quality. The decision of a region or a voxel belonging to a tumor is therefore fuzzy. Since the characteristics of the tumor are different from each image type, we have to model three fuzzy sets using different membership function.

According to the description of radiology experts, the characteristics of brain tumor on the three types of MR images can be presented in Table 1 .

\begin{tabular}{|c|c|c|c|}
\hline \multirow{2}{*}{ Tissues } & \multicolumn{3}{|c|}{ Signal Intensity } \\
\cline { 2 - 4 } & T1 & T2 & PD \\
\hline tumor & - & ++ & ++ \\
\hline edema & -+ & + & + \\
\hline CSF & -- & ++ & +- \\
\hline
\end{tabular}

Table 1: Intensity characteristic of brain tissue on MR images

The symbol "+" presents a hyper-signal, it means the image intensity is very bright. While "-" presents a hypo-signal, the intensity is very dark. The symbol "-+" presents the signal intensity is brighter than hypo-signal, and "+-" presents it is darker than hyper-signal. ".." presents the signal intensity is darker than the hypo-signal, and "++" presents it is brighter than the hyper-signal.

In Table 1, we can find that the signal intensity of tumor is neither very bright nor very dark in T1, but it is the brightest in $\mathrm{T} 2$ and $\mathrm{PD}$. Let us define the membership functions of tumor set corresponding to $\mathrm{T}_{\mathrm{T} 1}, \mathrm{~T}_{\mathrm{T} 2}$ and $\mathrm{T}_{\mathrm{PD}}-m f T_{\mathrm{T} 1}, m f T_{\mathrm{T} 2}$ and $m f T_{\mathrm{PD}}$ respectively. According to Table 1 , we can model the membership functions of tumor set for $\mathrm{T}_{\mathrm{Tl}}, \mathrm{T}_{\mathrm{T} 2}$, and $\mathrm{T}_{\mathrm{PD}}$ using the formula (1), (2) and (3) respectively.

$$
\begin{gathered}
m f T_{T 1}(x)=e^{-k(x-a)^{2}} \\
m f T_{T 2}(x)=0.5+0.5 \sin \left(\left(x-\frac{a_{1}+a_{2}}{2}\right)\left(\frac{\pi}{a_{2}-a_{1}}\right)\right), \quad a_{1}<x \leq a_{2}
\end{gathered}
$$




$$
m f T_{P D}(x)=\left\{\begin{array}{lc}
\frac{1}{2}\left(\frac{x-b_{1}}{b_{1}-b_{2}}\right)^{2}, & b_{1}<x \leq b_{2} \\
1-\frac{1}{2}\left(\frac{x-c}{c-b_{2}}\right)^{2}, & b_{2}<x \leq c \\
1, & x>c
\end{array}\right.
$$

where the variable $x$ is the membership value. The parameters $k, a, a_{1}, a_{2}, b_{1}, b_{2}$ and $\mathrm{c}$ are defined by the statistic values of each MR volume. These functions are shown in figure 1.

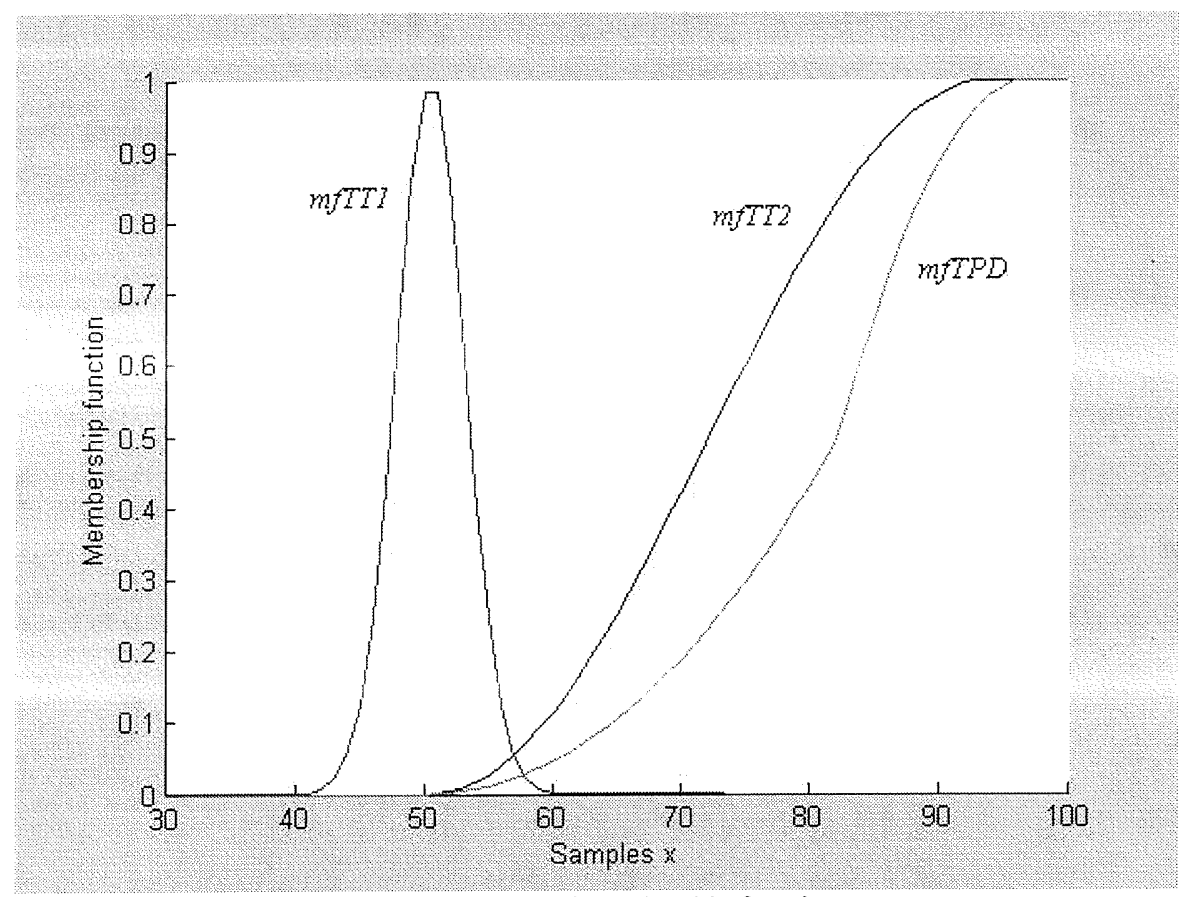

Figure 1: Curve of membership function.

\section{TUMOR EXTRACTION USING FUZZY INFORMATION FUSION}

When the radiology experts decide the brain tumor area on MR images, they check all three types T1, T2 and PD. This information fusion process helps the experts to remove the ambiguities of each image type in order to give a good diagnostic.

Since the three fuzzy sets corresponding to the three images types are known (see above), we can define a fuzzy set $T$ that is used to characterize the brain tumor area. The fuzzy set $T$ has relationships with $T_{T 1}, T_{T 2}$ and $T_{P D}$, written as follows:

$$
T \subset T_{T 1}
$$




$$
\begin{aligned}
& T \subset T_{T 2} \\
& T \subset T_{P D}
\end{aligned}
$$

$\mathrm{T}$ is therefore contained in the intersection of $\mathrm{T}_{\mathrm{T} 1}, \mathrm{~T}_{\mathrm{T} 2}$ and $\mathrm{T}_{\mathrm{PD}}$

$$
T \subseteq T_{T 1} \cap T_{T 2} \cap T_{P D}
$$

It means that the fuzzy set $T$ is the association of intensity information of fuzzy set $T_{T 1}, T_{T 2}$ and $T_{P D}$. We call the information association like this as fuzzy information fusion. The fuzzy set $T$ found on fuzzy information fusion will be characterized by an association membership function $m f T$. According to (4), we have

$$
m f T(x)=\min \left\{m f T_{T 1}(x), m f T_{T 2}(x), m f T_{P D}(x)\right\}
$$

where $x$ is a given threshold.

\section{EXPERMENTS AND VALIDATION}

The MR images used in our experiments have the following specifications: the number of slice on T1 is 124 , the slice thickness is $1.5 \mathrm{~mm}$, and the pixel size is $(0.9375 \times 0.9375) \mathrm{mm}^{2}$. The number of slice on original T2 and PD is 20 , the slice thickness is $5 \mathrm{~mm}$, and the pixel size is $0.46875 \times 0.46875 \mathrm{~mm}^{2}$.

We show here our results step by step.

\section{Step 1 Registration}

There are many registration methods. We selected the FLIRT (FMRIB's Linear Image Registration Tool), a linear interand intra-modal registration, proposed by Oxford Center for Functional Magnetic Resonance Imaging of the Brain (FMTIB). Figure 2 is one example of the result of registration using FLIRT. After registration, the voxel size on T2 related to $\mathrm{T} 2$ and $\mathrm{PD}_{\text {reg }}$ related to $\mathrm{PD}$ is equal to that on $\mathrm{T} 1,(0.9375 \times 0.9375 \times 1.5) \mathrm{mm}^{3}$ with 124 slices.

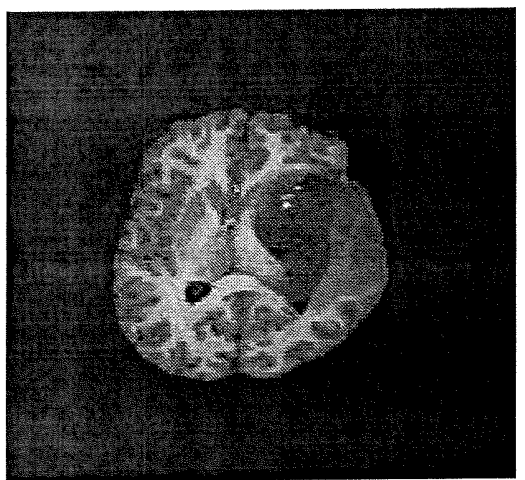

T1 weighted image

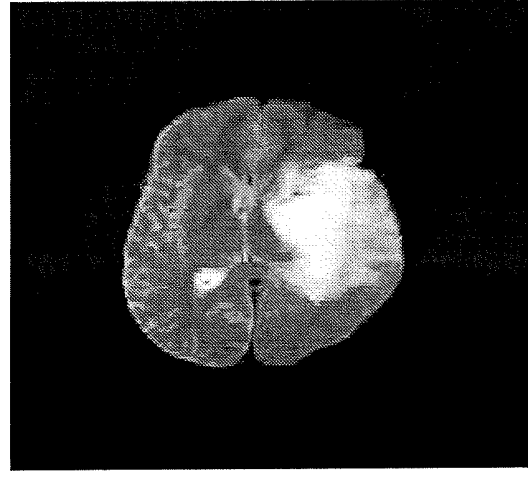

T2 weighted image after registration

Figure 2: An example of registration.

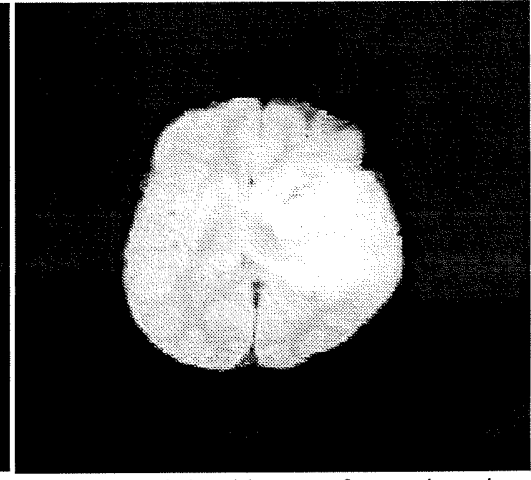

PD weighted image after registration

\section{Step 2 Brain tissue segmentation on $\mathrm{T} 1$}

[6] presents a brain tissue segmentation method using fuzzy Markovian Random Fields. The brain is classed into 11 classes in which there three pure classes representing the three main brain tissues, and 9 mixclasses representing different 
mixing of the three tissues (Figure 3). The brightest intensity and the darkest represent the white matter and the CSF respectively, the gray intensity represents the gray matter. The others intensities represent the mixclasses.

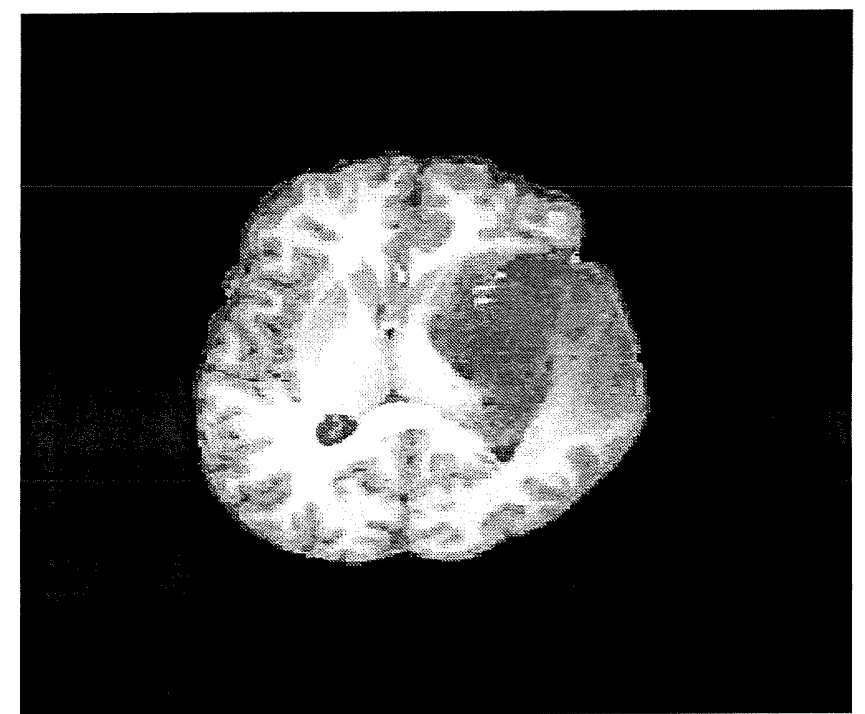

Figure 3: One slice of the segmented 11 classes on T1 weighted MR image

\section{Step 3 Fuzzy information modelization}

The classes obtained after the step two are used to extract the fusion region on $\mathrm{T} 1, \mathrm{~T}_{2}$ reg and $\mathrm{PD}_{\text {reg. }}$. In each class, labeled regions are considered as a basic element of fuzzy set for fusion. The statistical values of the classes in each image type are calculated and used to define the parameters of the membership functions (1), (2) and (3).

\section{Step 4 Brain tumor extraction}

Since the three fuzzy sets with membership functions (1), (2) and (3) are known, the brain tumor area, a fuzzy set $T$, can be obtained by using (5). Figure 4 (a) is the result with a threshold of membership value 0.9 . This result is compared to that obtained by a radiology expert, shown in figure 4 (b). There is $2 \%$ of false negative and $1.3 \%$ of false positive.

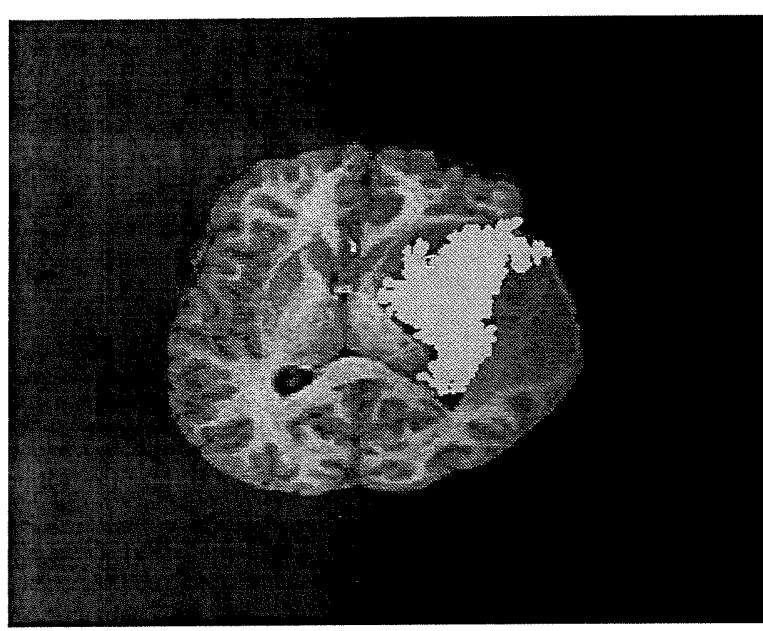

(a)

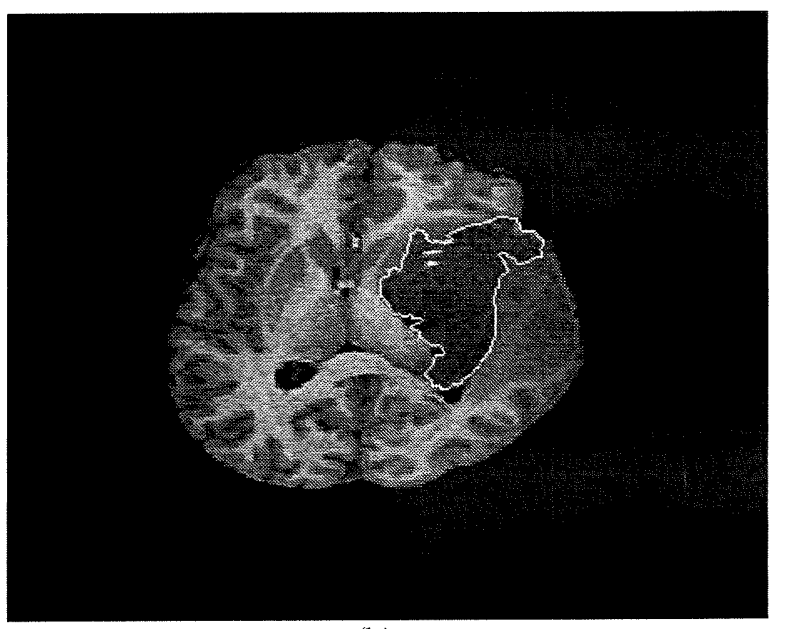

(b)

Figure 4: Comparison of results. (a) Automatic result. (b) Manual result of a radiology expert diagnosis 


\section{CONCLUSION}

We have presented a method to extract the brain tumor from mutispectral MR images. There are two fundamental premises on which this method is based on: (a) The proposed method is a general method to extract automatically human brain tumor. The conception of fuzzy information fusion, proposed in this paper, is universal. It can be used to solve any incomation extraction problem just by adapting the membership functions. (b) The proposed fuzzy membership functions were modeled according to characteristic descriptions provided by the experts for each image type, therefore allowing to well exploiter the a priori knowledge. The choice of the threshold of membership value in the step of the fusion influences directly the result. The automatic choice of the threshold is our future work.

\section{REFERENCES}

[1]. V.Barra and J. Boire, "Automatic Segmentation of Subcortical Brain Structures in MR Images Using Information Fusion", IEEE Transactions on Medical Imaging 20, pp. 549-558, July 2001.

[2]. M. Clark, L. Hall, D. Goldgof and R.Velthuizen et al., "Automatic Tumor Segmentation Using Knowledge-Based Techniques", IEEE Transactions on Medical Imaging 17, pp. 187-201, April 1998.

[3]. S. Sobottka, R.Steinmeier and B. Beuthien-Baumann et al., "Evaluation of automatic multinodality fusion technique of PET and MRI/CT images for computer assisted brain tumor surgery", International Congress Series, Volume 1230, pp. 261-267, June, 2001

[4]. J. Udupa, L. Wei, and S. Samarasekera et al., "Multiple Sclerosis Lesion Quantification using Fuzzy-Connectedness Principles", IEEE Transactions on Medical Imaging 16(5), pp. 598-609, 1997

[5]. M. Jenkinson and S. Smith, "Optimisation in Robust Linear Registration of Brain Images", FMRIB Technical Report TROOMJ2.

[6]. S.Ruan, B. Muretti, J. Fadili and D. Bloyet, "Segmentation of Magnetic Resonance Images using Fuzzy Markov Random Fields", Proceedings of 2001 IEEE International Conference on Image Processing (ICIP 2001), pp. 1051-1054, Thessaloniki, Greece, October 7-10, 2001. 